Meta

Journal des traducteurs

Translators' Journal

\title{
La terminologie : méthodes de définition des synonymes
}

\section{Mary-Louise Kearney}

Volume 26, numéro 3, septembre 1981

URI : https://id.erudit.org/iderudit/003297ar

DOI : https://doi.org/10.7202/003297ar

Aller au sommaire du numéro

Éditeur(s)

Les Presses de l'Université de Montréal

ISSN

0026-0452 (imprimé)

1492-1421 (numérique)

Découvrir la revue

Citer cet article

Kearney, M.-L. (1981). La terminologie : méthodes de définition des synonymes. Meta, 26(3), 284-287. https://doi.org/10.7202/003297ar d'utilisation que vous pouvez consulter en ligne.

https://apropos.erudit.org/fr/usagers/politique-dutilisation/ 


\section{LA TERMINOLOGIE : MÉTHODES DE DÉFINITION DES SYNONYMES}

La synonymie constitue un problème important dans le domaine de la terminologie. Puisque le terminologue vise une nomenclature bien définie, qui comprend tous les termes clés, pertinents à une activité ou à un métier spécifique, les synonymes méritent une attention spéciale. Comme Bruno de Bessé observe :

La synonymie est rare, on ne trouve que peu ou pas de synonymie dans

les unités complexes. Quand il y a synonymie apparente, il y a toujours un élément situationnel qui empêche une synonymie absolue ${ }^{1}$.

Ainsi le quasi-synonyme intéresse le terminologue parce que sa définition est souvent très difficile à préciser. Pour l'effectuer, le terminologue doit se servir des critères linguistiques et extra-linguistiques.

Le but de notre projet était d'établir la nomenclature de l'industrie du Pursang en France. C'est-à-dire, nous cherchions à ranger les termes employés dans le cadre du commerce international de cette industrie.

Après avoir dépouillé un corpus composé de documents authentiques et de presse, se rapportant à cette industrie, nous sommes arrivés à établir une nomenclature finale de 1180 termes, distribués d'après les divers secteurs de ce domaine. Le schéma suivant démontre la fréquence de la synonymie :

\begin{tabular}{lrccccc}
\hline & & Élevage & Identification & Ventes & Administration & Performance \\
\hline Synonymes & 114 & 30 & 2 & 4 & 9 & 69 \\
Quasi-synonymes & 7 & 1 & -2 & 2 & 7 & 4 \\
Total & 121 & 31 & 2 & 6 & 9 & 73 \\
\hline
\end{tabular}

1. Bruno de Bessé (1973): "Observation de la Synonymie dans la terminologie de la Métallurgie », La normalisation linguistique, Québec, p. 47. 
Pour que le lexique soit utile et heuristique à un public de lecteurs professionnels, qui travaillent dans cette industrie, il fallait bien définir les synonymes. Selon les dictionnaires qui portent sur ce domaine, nous avons découvert une définition pour la plupart des termes. Donc, la synonymie était évidente en raison de nombreuses définitions identiques :

E.G. Être en queue

Fermer la marche

Anglais : to bring up the rear

Occuper le dernier rang

Définition: décrit le concurrent qui court derrière le lot.
E.G. La conformation
Anglais : the conformation

Le modèle:

Définition: se dit de l'ensemble général du cheval: ses membres et son apparence.

E.G. L'allure

Anglais : the pace

Le train

Définition: se dit de la vitesse de déplacement du cheval.

Pour mieux présenter ces synonymes à nos lecteurs, nous avons envoyé chaque groupe à un comité d'experts du domaine même. Ils ont exprimé leur avis concernant ces synonymes pour que lecteur sache le statut de chaque terme. De nombreux termes étaient interchangeables:

E.G. Etre peinture anglaise

Anglais : to be lean

Etre racing-like

De l'autre côté, il était évident qu'un terme particulier était souvent préféré pour une raison spécifique :

E.G. L'amélioration de la race

L'amélioration génétique (zootechnique)

E.G. Le père

L'auteur (presse)

E.G. Solliciter

Demander (populaire)

E.G. Le premier garçon

Le head-lad (terme emprunté)

\author{
Anglais : the improvement of the breed \\ Anglais : the sire \\ Anglais : to set alight \\ Anglais: the head lad
}

Malgré cette gamme de raisons de préférence, le terminologue devrait noter chaque détail. De cette façon, son public sera mieux renseigné sur le meilleur usage de chaque terme. Donc, le terminologue doit consulter et le dictionnaire et un comité d'experts pour bien regrouper les synonymes de la nomenclature.

\title{
LES QUASI-SYNONYMES
}

Les quasi-synonymes deviennent un peu plus difficiles à définir. Nous avons trouvé sept quasi-synonymes proprement dits: c'est-à-dire, des termes qui sont presque identiques du point de vue de sens. Chacun d'eux possède un trait spécial et par conséquent, ils ne sont pas interchangeables.

Nous avions dépouillé ces termes d'après le corpus de presse, milieu qui tient à employer un vocabulaire varié. En étudiant le contexte de leur usage, le caractère spécifique de chaque terme s'est révélé. Une fois que nous avions trouvé une définition générale, nous avons adopté le critère du trait pertinent. Cette approche a été proposé par A.J. Greimas comme une méthode de distinguer le statut individuel de chaque terme (Greimas 1976, p. 18). 
E.G. La descendance (générale)

Anglais : the offspring

Les descendants

La production (directe)

Définition: L'ensemble des produits qui peuvent remonter à un auteur.

\begin{tabular}{lccc} 
& Pedigree & Sexe & Parenté \\
\hline Descendance & - & $\mathrm{m}+\mathrm{f}$ & générale \\
Descendants & - & $\mathrm{m}+\mathrm{f}$ & générale \\
Production & - & $\mathrm{m}+\mathrm{f}$ & directe \\
\hline
\end{tabular}

E.G. La qualité (naissance)

La trempe (résistance)

Anglais : quality

Définition: décrit la caractéristique exceptionnelle de pedigree ou de fond du cheval de course.

\begin{tabular}{lcc} 
& Naissance & Résistance \\
\hline Qualité & - & \\
Trempe & & - \\
\hline
\end{tabular}

En principe, de tels termes possèdent la même définition. Cependant, il existe une petite différence entre chaque mot. Seul un expert était capable de confirmer ce trait clé, qui se rapporte à l'analyse sémique de Greimas. En raison de leur connaissance parfaite de leur domaine, ces spécialistes emploient constamment les concepts clés. Donc ils peuvent distinguer le sens particulier et l'usage de chacun immédiatement. Le terminologue doit consulter un comité de ces experts pour que la nomenclature soit exacte et utile à son public d'usagers professionnels. En effet, une consultation régulière est souhaitable pour faciliter la tâche du terminologue.

\section{CONCLUSION}

Pour conclure, il est évident que deux facteurs sont nécessaires pour que la synonymie soit bien étudiée :

$1^{\circ}$ le critère linguistique: une bonne définition de base

$2^{\circ}$ le critère extra-linguistique : l'opinion des spécialistes du domaine visé pour distinguer les traits importants des quasi-synonymes.

Selon Pierre Auger et Louis-Jean Rousseau, ces experts sont «...des témoins clés de la langue, du milieu observé» (Auger et Rousseau 1978, p. 21). Donc, le terminologue doit accepter leurs conseils.

Aujourd'hui, le but de la terminologie est de faciliter la communication internationale dans toute activité spécialisée. Ainsi, le terminologue doit se servir d'une qualité de critères pour réaliser un travail de meilleure qualité.

MARY-Louise KeARNeY

\section{BIBLIOGRAPHIE}

ASSOCIATION FRANÇAISE DE TERMINOLOGIE (1977): Terminologies 76, Paris, La Maison du Dictionnaire, $1102 \mathrm{p}$. 
AUGER, P. et L.-J. ROUSSEAU (1978): Méthodologie de la recherche terminologique, Québec, Office de la langue française, $80 \mathrm{p}$.

FELBER, Helmut (1977) : «Fundamentals of a General Theory of Terminology», Vienna, INFOTERM, $16 \mathrm{p}$.

GREIMAS, A.J. (1976): Sémiotique et Sciences sociales, Paris, Éditions du Seuil, 215 p.

OFFICE DE LA LANGUE FRANÇAISE (1974): "La Normalisation linguistique», Actes $d u$ colloque international de terminologie, Québec, O.L.F., 253 p.

SANDERS, T.R.B., ed. (1972): The Aims and Principles of Standardization, Genève, I.S.O., $115 \mathrm{p}$. 